\title{
Advanced Air Mobility: Research Directions
}

This paper was downloaded from TechRxiv (https://www.techrxiv.org).

\section{LICENSE}

$\mathrm{CCO}$

SUBMISSION DATE / POSTED DATE

06-10-2021 / 14-10-2021

CITATION

Namuduri, Kamesh (2021): Advanced Air Mobility: Research Directions. TechRxiv. Preprint. https://doi.org/10.36227/techrxiv.16746193.v1

$\mathrm{DOI}$

10.36227/techrxiv.16746193.v1 


\title{
Advanced Air Mobility: Research Directions
}

\author{
Kamesh Namuduri*, Uwe-Carsten Fiebig*, David W. Matolak*, Ismail Guvenc * , K. V. S. Hari *, and \\ Helka-Liina Määttänen *
}

Abstract-Advanced Air Mobility (AAM) is a newly emerging industry focus, as well as a research and development discipline. Innovations and technologies resulting from AAM will change the way that we move cargo and people in and around cities. Industry is moving fast with excitement to deploy AAM solutions. However, there are multiple technical challenges that need to be overcome before AAM becomes a reality. This article takes a closer look at the technology readiness level of AAM solutions, identifies open research problems and directions to address them.

Index Terms_-Advanced Air Mobility

\section{INTRODUCTION}

Advanced air mobility (AAM) will add a new dimension of mobility to our lifestyle - unmanned transportation of people and goods in and around cities [1]. Once AAM becomes a reality, hundreds or even thousands of Unmanned Aircraft Systems (UAS) will be flying in our neighborhoods. Industry is moving with much enthusiasm, pushing aviation authorities to establish the rules of engagement. How far are we from the AAM solutions, including air taxi and air ambulance? This article highlights several key research problems and ways to address them.

\section{A. AAM Architecture}

Originally called Urban Air Mobility (UAM), AAM (Fig. 1) is an evolution from its predecessor UAS Traffic Management System (UTM). While UTM is designed for small UAS flying at or below $400 \mathrm{ft}$. Above Ground Level (AGL), AAM includes larger aircraft carrying people and cargo at altitudes between 500-2000 ft AGL. AAM platforms include newer aircraft designs with vertical takeoff and landing (VToL) capabilities. A key subsystem in the AAM architecture [2] is the Provider of Services for UAM (PSU), which will provide services to support operations planning, flight intent sharing, strategic and tactical deconfliction, and airspace management functions. PSUs will exchange information with other PSUs via a network that enables safe, efficient operation within the AAM corridors. PSUs make use of Discovery and Synchronization Service (DSS) to identify active areas where other aircraft are flying, as well as UAS Volume Restrictions (UVRs), representing areas that need to be avoided due to hazards or other type of restrictions, both permanent and temporary. The AAM architecture will include Supplementary Data Service Providers (SDSPs) that provide services such as weather and Fight Management Information System (FIMS) that are used to manage manned aviation. ASTM specifications are being followed for data exchange protocols between one subsystem to another within the AAM ecosystem.

\section{B. Contributions}

While there are several technical and societal barriers that need to be addressed before AAM services such as air taxis and air ambulances can be deployed for real-world applications, this article highlights some of the most critical challenges to which academic researchers can contribute. These include: (1) Air Corridors; (2) Air-to-Air Communications; (3) 3GPP Support for Navigation; and (4) Detect and Avoid / Collision Avoidance. Few other areas of research including (5) Navigation in GPS-denied environments; (6) Noise mitigation; (7) Security and Privacy; and (8) Community Engagement are briefly discussed. This list is not exhaustive, but represents the critical subset of challenges. Although some of these problems may have equivalent solutions in terrestrial systems or manned aviation systems, they are not always directly portable to the AAM ecosystem.

\section{AIR CORRIDORS}

Air corridors are 3D volumes of airspace reserved for UAS for AAM traffic. Air corridor design specifications are specific to each country and are defined by the respective federal aviation authorities. In the United States, Federal Aviation Authority (FAA) defines air corridors in class B, C, or D airspace. FAA also defines the expected performing requirements of a UAS flying in an air corridor.

The design of air corridors follows the overall goal to provide efficient and safe operation of UAS, respecting UVRs and environmental issues. If only few UAS are airborne the control of UAS and their safe operation is relatively easy. However, as their number is considerably growing an appropriate air corridor concept has to be developed, which set the rules for the choice of flight trajectories: on the one extreme trajectories may not be subject to any restrictions, an example is that UAS fly along the shortest path between take-off and landing locations. The other extreme is that only predefined 3D flight routes are allowed, an example is that UAS must follow the layout of streets. Still, there are numerous open questions and we like to mention a few: which air corridor concept is the safest one, requires the least amount of coordination for collision avoidance, provides the highest flexibility to cope with growing UAS traffic, guarantees the shortest flight times, allows for priority flights, can handle the bottleneck of locations where the rates of take-offs and landings are extremely high (like at a distribution warehouse), is robust when hazards emerge.

A possible air corridor concept is illustrated in Fig. 2 consisting of air corridors with three layers. This design is shown for visualization purposes only and is not approved or standardized by any authority. In this design, the top and bottom layers contain one-directional tracks or skylanes. The middle layer contains intersections (roundabouts) for the AAM aircraft to change the direction of travel. For example, if a Southbound UAS needs to turn East, it will lower down from layer one to layer two, takes a quarter turn in the roundabout, climbs down to layer three, merges the Eastbound skylane, and continues its travel. The design of such air corridors, traffic rules in the air corridors, safety requirements, and performance specifications are still evolving. Air space design concepts such as the geofence [3] are currently being considered by various research groups. 


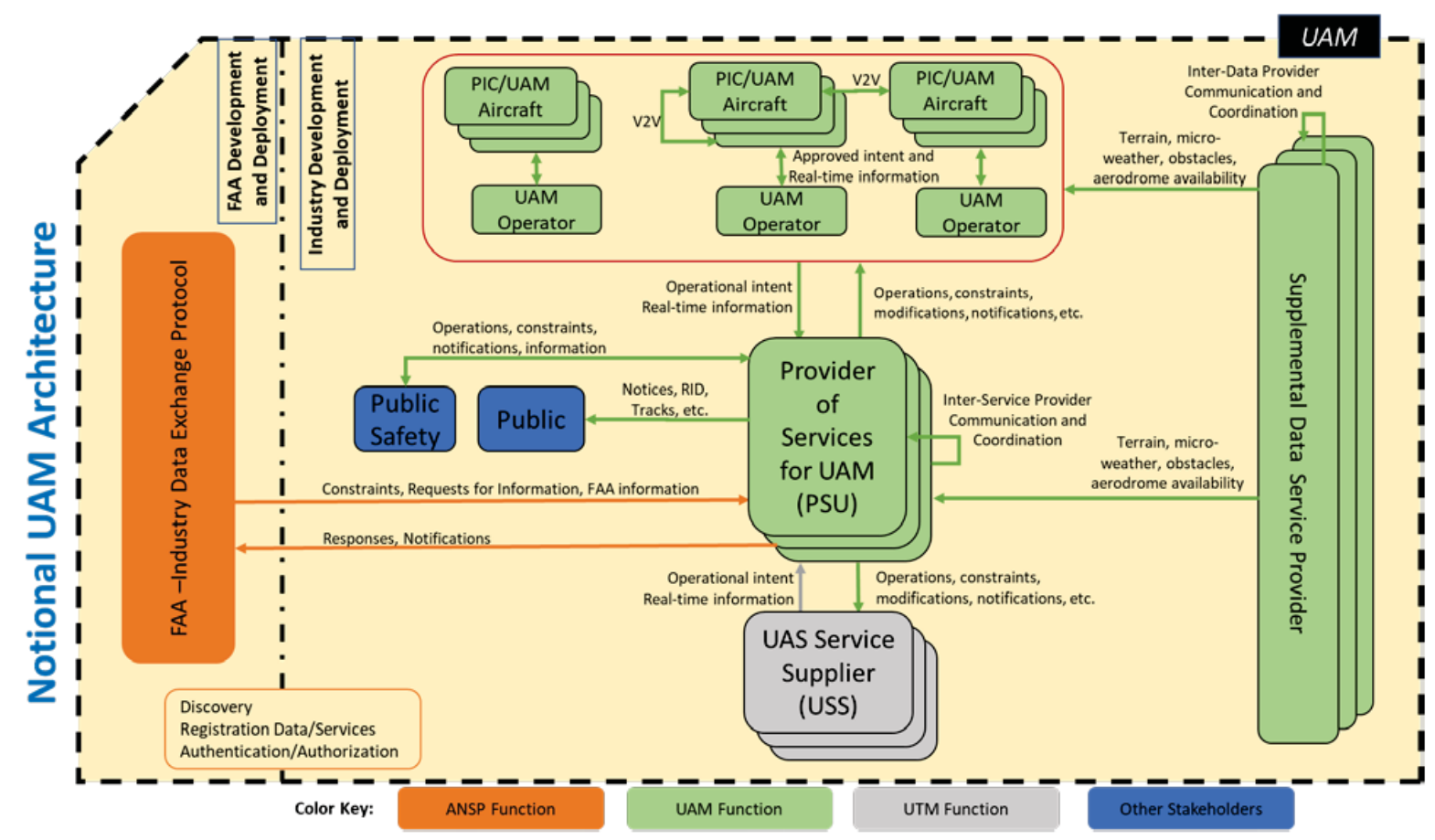

Figure 1: AAM UAM Architecture, Courtesy NASA

There is a need for finding a substitute for traffic lights in air corridors. The most obvious choice for this substitution is vehicle to vehicle (V2V) communications among the UAS. A simple use case for $\mathrm{V} 2 \mathrm{~V}$ communications in air corridors is illustrated in Fig. 3. It depicts a scenario in which normal flight operations are taking place in a skylane and suddenly one of the vehicles detects an airspace hazard (obstacle such as a solid cloud). In this scenario, vehicle A which detected the hazard acts first. It needs to avoid the obstacle and share this information to the following vehicles, $\mathrm{B}$ and $\mathrm{C}$ in real-time. Further, depending on the estimated time that it takes for the airspace hazard to dissipate, information needs to be percolated to the ground control station (GCS) to avoid potential congestion in the skylane. Standardization efforts for V2V communication protocols, use cases, spectrum needs, and security requirements are currently taking place in the AAM community. Section III outlines the fundamental research needed for developing efficient V2V communication strategies.

$\mathrm{V} 2 \mathrm{~V}$ communications can be combined with the principles of Radar systems to develop Dual Function Radar Communication (DFRC) systems where radar waveforms can be designed to carry information in order to communicate with other vehicles in the vicinity.

\section{AIR-TO-AIR COMMUNICATIONS}

Air to air (AA) communications, like air-to-ground (AG) communications, has a history nearly as long as aviation itself. As with terrestrial settings, multiple network topologies (e.g., mesh, relay, star, etc.) can be employed. A primary difference from terrestrial networks arises from high aircraft speeds: AA network topologies can change rapidly. Until air traffic densities become much larger than presently envisioned for AAM applications, AA communications will likely serve a supporting role to primary AG communication links, whereas navigation, and in particular surveillance, will rely more on AA signaling. Individual aircraft surveillance is usually termed detect and avoid (DAA). For all three functions-communications, navigation, and surveillance (CNS)-reliability at the lower layers of the communications protocol stack is critical. Hence in this section we focus on AA signaling at the physical (PHY) and data link layers (DLL).

When link distances are on the order of altitudes or smaller, the AA channel is very close to a free-space channel. The primary obstacle that affects the AA channel is the earth surface, and as link distances increase, reflections from the earth surface become the next significant channel component in addition to the line-ofsight (LOS) component [4]. For lower aircraft altitudes, AA link range will decrease, since radio line of sight over the curved earth is proportional to the square-root of altitude. In addition, the probability of obstruction of the AA link increases as altitude decreases.

In the best of circumstances when aircraft are well above obstacles, one can approximate the AA channel as a pure free-space channel, with propagation path loss proportional to the reciprocal of the square of link distance $d$. Proximity to the earth, and to earth-based objects, can substantially alter this first-order model. For links between aircraft that are not too close to ground, and at approximately the same altitude, this $1 / d^{2}$ free-space path loss model allows large link ranges in comparison to terrestrial settings, where often a $1 / d^{3}$ or $1 / d^{4}$ attenuation relation holds.

The simplest enhancement to the free-space channel model is to account for the presence of the earth. If link distances are short enough, a flat-earth model may suffice; this will depend on link geometry, aircraft altitude, and relative terrain roughness. Including an earth-surface reflection makes this a two-ray channel, for which many good models exist [5].

At altitudes low enough to allow significant reflections from terrestrial obstacles, additional multipath components (MPCs) may 


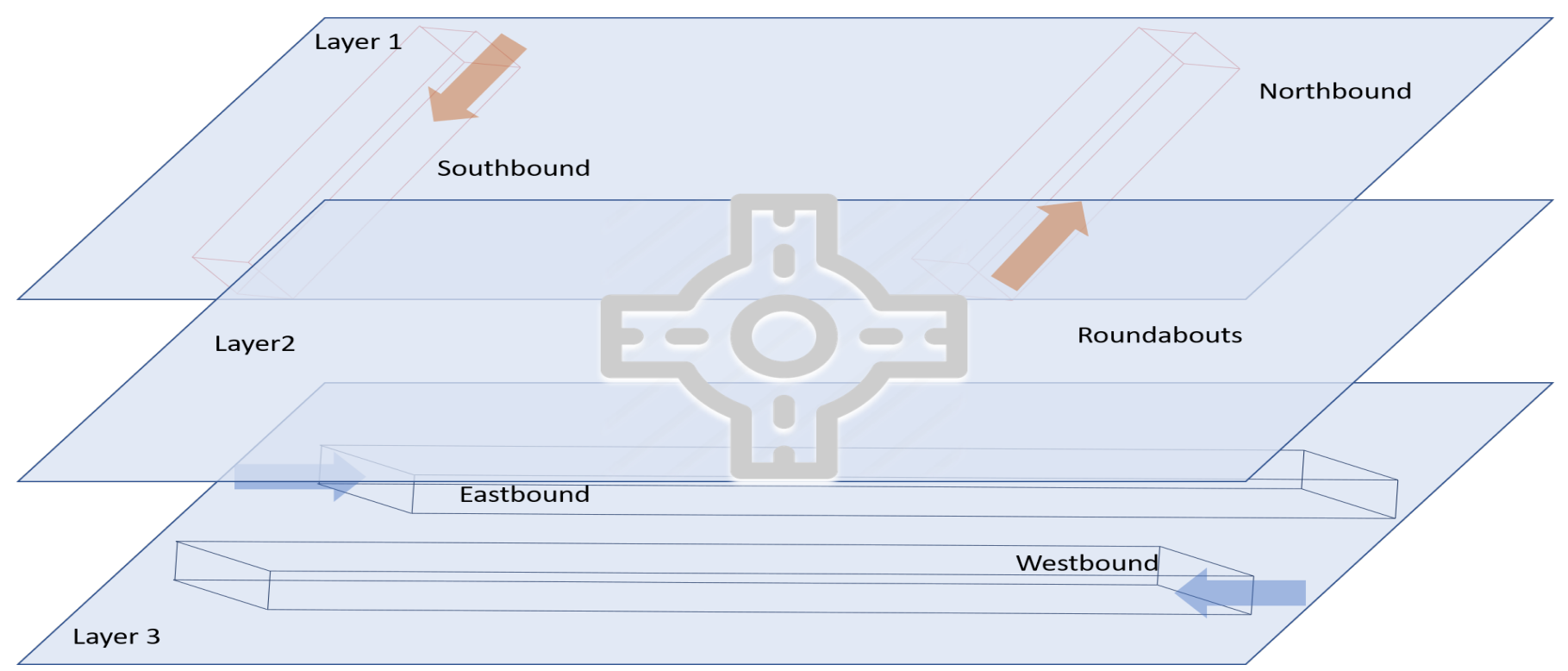

Figure 2: Illustration of an air corridor: author's visualization only

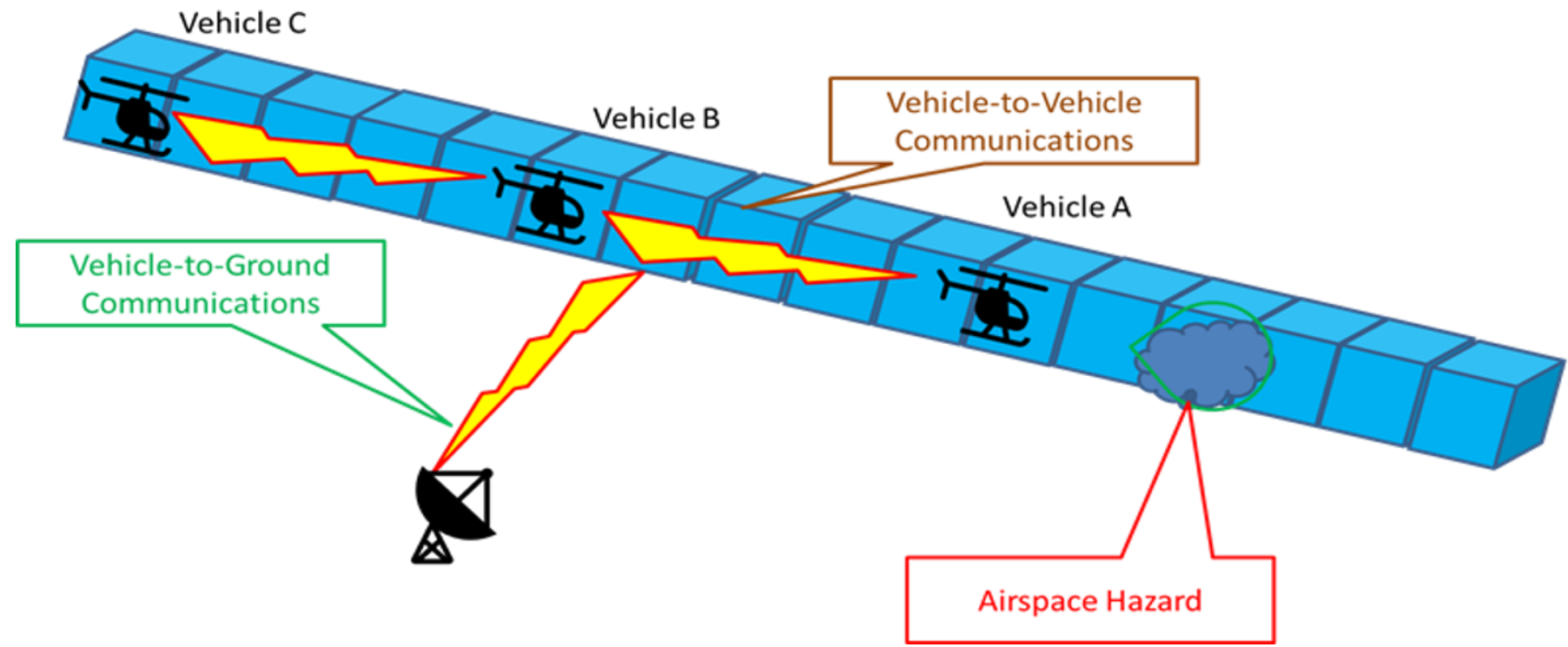

Figure 3: The need for V2V communications in air corridors: A use case

be present. These can occur at relatively large values of delay, and with aircraft motion, will tend to be intermittent, as found for the AG channel [6].

After the link geometry and environment, antenna characteristics are another primary factor affecting the physical-layer of AA links. For example, a well-designed simple monopole antenna will have its antenna pattern changed-sometimes drastically-when mounted on an aircraft. Mounting location is also important, since an antenna mounted on one side of the fuselage (e.g., the top) will typically suffer significant gain reduction if used to receive a signal coming from the direction of the other side of the fuselage (e.g., the bottom). Thus, multiple antennas distributed across the aircraft may be required if connectivity is truly required to be 3-dimensional.

Directional antennas suppress MPCs and can enable larger link ranges, but in a dynamic AA setting, antenna pointing and tracking can be challenging. For AAM applications, some of the most challenging cases will be in the near-urban and urban environments. In the nearurban case, flights will likely follow established air corridors well above buildings, hence AA channels will likely be well modeled by a two-ray channel, in which terrain cover type will determine the strength and nature of the reflection (specular or diffuse). Work on estimating and validating these air corridor channel models should be done for the appropriate frequency bands.

For the urban case, additional MPCs will be present from buildings that will also sometimes act as obstructions. Obstruction attenuation generally increases with frequency. To date, largely because of the difficulty of conducting flight tests over and within urban areas, most channel models for this case rely solely on analyses and simulations. Thus, actual experimental work on this topic would be very valuable.

A first wideband measurement campaign which aims at getting a deeper understand of signal propagation in urban AA environments has been conducted in 2019 [7]. Various flight scenarios have been 
assessed with the goal to measure the propagation conditions in challenging scenarios: E.g., two drones fly behind each other along building facades at close distance to the building, see Fig. 4, a drone lands in the inner courtyard of a building while another one flies around the building, two drones fly on a potential collision course towards the corner of a building. Inherently, LOS as well as non LOS situations have been assessed. First findings reveal that the channel exhibits a large variety of propagation phenomena encompassing single, double and triple reflections, diffraction, scattering, shadowing, and combinations of all. The evaluation is ongoing but already indicate that the AA channel for urban scenarios in which drones fly below rooftops is at least as complex as the car-to-car channel.

A future research goal is to identify critical propagation scenarios like the occurrence of strong reflections potentially arising from large window facades or long-lasting shadowing. Those scenarios are crucial for detect and avoid since they may either interrupt the AA communication link or result in an incorrect reception of messages. Although two potentially colliding drones may have many chances to receive respective messages from each other prior to the potential collision but it is important to understand to which extent the propagation medium contributes to communication outages. Furthermore, there are events in which a collision course occurs unexpectedly like a mechanical failure or a sudden gust. Neighboring UAS have to fast and reliably receive information about the event. Therefore, a deep understanding about the channel conditions is very valuable.

For actual signaling formats, this will depend on applications. There may be some messages that have very stringent reliability requirements, as well as latency requirements. The use of the cellular community's ultra-reliable low-latency communications (URLLC) is hence worthy of consideration for these cases. Otherwise, it is likely that AA links will employ the same signaling schemes as AG links. Contending schemes include 5G cellular, VHF Digital Link (VDL), Lband Digital Aeronautical Communications (LDACS), Aeronautical Mobile Airport Communication System (AeroMACS), as well as others, such as military systems (Link 16, Common Data Link), and schemes still under development.

\section{3GPP SUPPORT FOR NAVIGATION}

In Release-15, 3GPP conducted a study in order to investigate the ability of the LTE network to provide connectivity for low altitude UAS. The study defined performance requirements for both command and control as well as for application data for the UAS. From 3GPP perspective, both categories are user plane traffic. For command and control, the latency and bit error rate were concluded to be more important than data rate which then again is more crucial to application data, especially with a video use case. Further, channel models and typical scenarios were defined for studying the potential issues and potential solutions to be addressed later in the work item phase. The outcome of the study was documented in the 3GPP technical report [8] including also field measurement results. In [9], the authors present a related study and findings of UAS operation in cellular networks.

The Release 15 LTE work item, approved in [10], specified the key features identified during the study item phase to enhance the UAS operation in cellular networks. One of these features is height reporting when a UAS crosses a height threshold. Multiple height thresholds can be configured for a UAS, e.g. $50 \mathrm{~m}, 100 \mathrm{~m}$ and $250 \mathrm{~m}$. The report includes height, location (3D) as well as horizontal and vertical speed. Another specified feature is reporting of the signal strength measured at the UAS when $N$ neighboring base station's signal strength is above a threshold. This, together with the height reporting, is used for both interference detection as well as flying mode detection. One key feature is core network signalling for subscription-based identification in order for the network to verify whether a mobile device can be served as a UAS in the network. Additionally, support for signalling flight path information from the UAS to the base station was added to the air interface signalling protocol. This includes network polling of list of waypoints (3D location), and time stamps if available. These enhancements are described in more detail in Chapter 6 in [11].

Currently, 3GPP is preparing for Release-18 which is planned to be started in Q2 of 2022. Specifying enhancements for UAS for $5 \mathrm{G}$ NR networks is one of candidates to be considered further and the current consensus for the content is aligned with the work item proposal in [12]. The scope of the potential work item includes introduce corresponding support for NR $5 \mathrm{G}$ as was specified for LTE, support for drone remote identification broadcasting over PC5 and additionally further enhancements for mobility and RRM.

The FAA has issued it's ruling on UAS identification and two methods are expected based on the class of drone. One is network tracking where location information of the UAS is conveyed back to a UAS service supplier. As the 5G NR already supports location reporting from a mobile device, a UAS in this case, to the network, there is no need for further 3GPP specification effort to enable this identification method. Additionally, the height threshold based height reporting may be configured to include location of the UAS as well. Fig. 5 depicts the UAS broadcasting ID information. The other one is UAS broadcast ID where the device transmits its identification and owner info. Based on the class and usage of the drone one or both network based and UAS ID broadcasting may be required.

In order for $5 \mathrm{G} \mathrm{NR}$ and LTE to support the ID broadcasting, specification effort is needed. 3GPP enables a Device-to-Device (D2D) link, also defined as sidelink or PC5, which is part of the cellular eco-system for Cellular Intelligent Transport Systems (ITS). The PC5 link enables communication between other vehicular mobile devices or towards road side units. This provides framework for supporting UAS remote ID broadcasting. As an LTE base station may schedule an 5G NR UE and 5G NR BS may schedule an LTE UE, it is currently discussed to add both 5G NR and LTE support for this feature in Release 18.

Additionally, the topic of collision detection and avoidance has emerged during the Release 18 discussions and is proposed to be addressed during the 3GPP normative work. The PC5 supports already broadcasting messages for collision detection and avoidance (D/A) over sidelink and reuse of these traffic-safety messages should be the starting point. However, there may be additional enhancements that could benefit the specific UAS use case such as sharing some information in flight paths.

The Release 18 UAS discussions also include considering further enhancements for uplink beamforming and beam management in order to further control interference and to improve link quality with faster switching of uplink beams towards the BS. However, these suggestions need to be considered against the MIMO Release 17 enhancements that may already provide the needed level of 


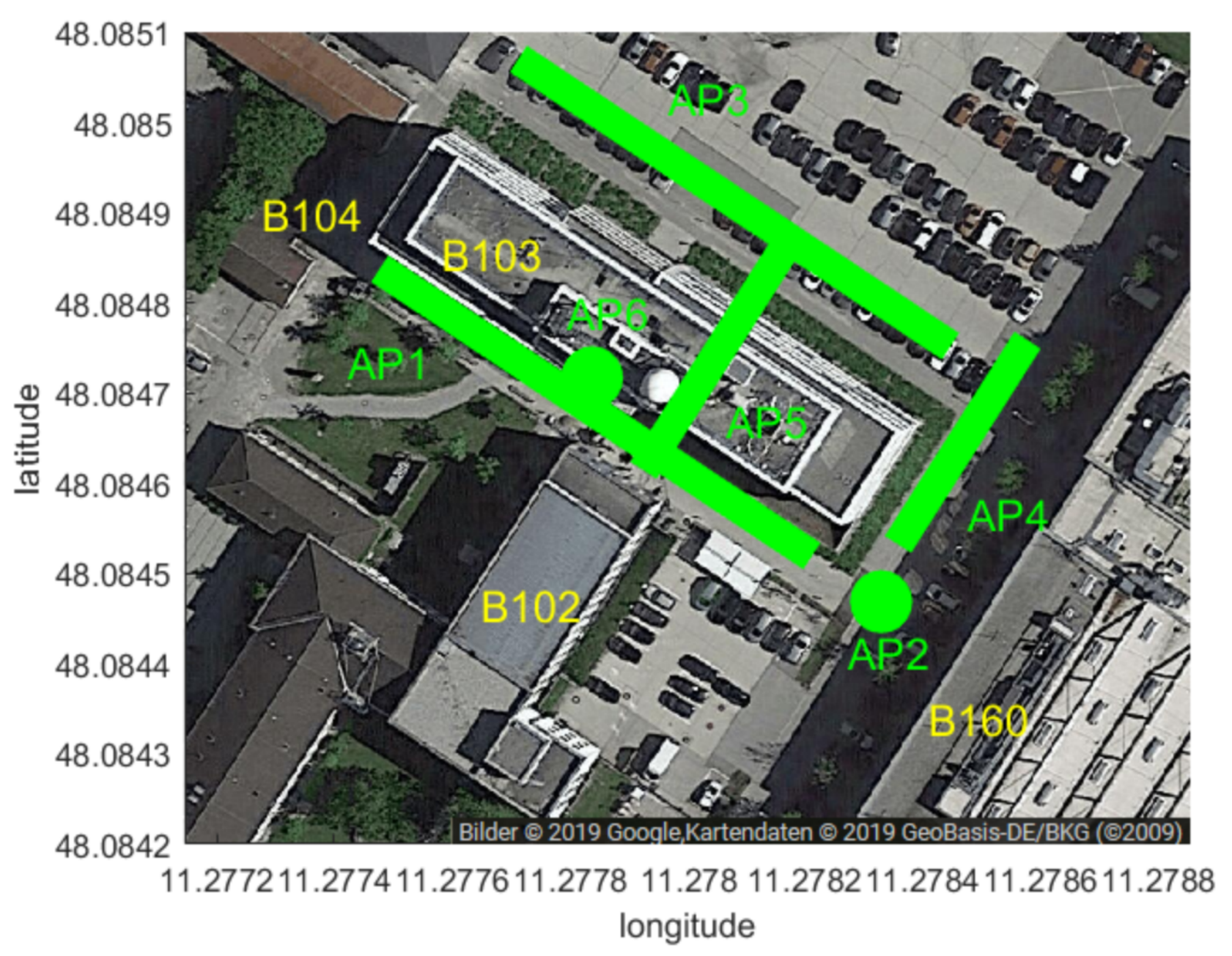

Figure 4: Illustration of some of the scenarios assessed in an AA propagation measurement campaign: two drones fly closely along the facade of building B103 behind each other; one drones flies along the North side of building B103 while the other drone flies along its South side; one drone is located at a balcony (AP6) of building B103 whereas the other drones flies over the rooftop of that building, etc.

improvements.

In order to enable better mobility performance, there are proposals to use and enhance a so called conditional handover (HO). In regular $\mathrm{HO}$, the mobile device starts connecting to the target cell immediately upon receiving the handover command. In case of conditional $\mathrm{HO}$, the mobile device receives a handover command with a condition, and when the condition fulfills, the devices starts connecting to the target cell. Currently, the conditional HO command may include up to 8 target cells and up to two conditions per target cell, and the conditions can be signal strength related only. For the UAS use case, additional conditions such as location or height may be considered. These could be used independently or in combination of a signal strength specific condition. For example, the UAS perfoms HO to a cell A when height is above a certain threshold and signal strength is above a certain threshold. The same cell may be also configured separately with only signal strength specific threshold. The device receiving both of these configurations will evaluate both and trigger $\mathrm{HO}$ based on which configuration is its conditions first full filled. In this way, the device may be guided to perform HO towards the cell at different signal strength conditions depending whether it is flying or not flying. This may be useful, as in a flying state the free space propagation becomes the dominating factor and the coverage map of the cells differs from the ground situation.

It should be noted the content of the Release 18 work is currently tentative and what is presented here is the current status of the discussions. There is largest consensus for importing LTE UAS functionality to NR as well as to specify drone remote ID broadcasting over PC5 to fulfill FAA requirement.

\section{DETECT AND AVOID}

Detect and avoid (DAA) is one of the most crucial components in AAM. Due to the large number of UAS, detect and avoid does 


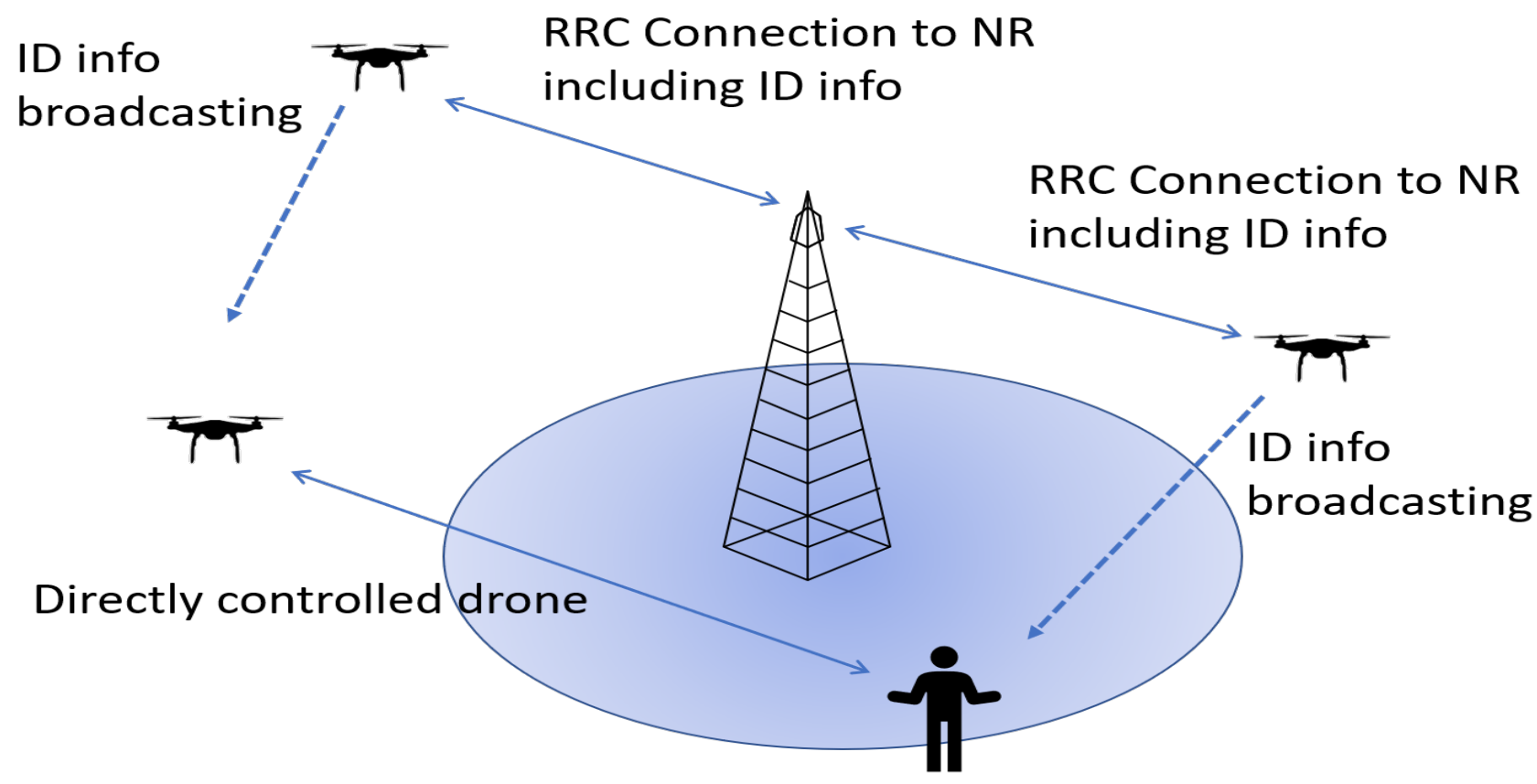

\section{Public officer reading ID info of both UAS connected to NR network}

Figure 5: Illustration of network based on direct UAS indentification. The two UAS are RRC connected and network is able to identify and locate the drones. Both drones are also configured to broadcast ID info which may include both device ID as well as owner information. Local officer may read the ID info directly with a handheld device or use a drone to read the info.

work reliably any more with humans in the loop but has to operate fully autonomously and has to be tailored to the air corridor concept, see Section II. Furthermore, when no planning of flight trajectories prior to take-off against potential collision courses is carried out, the DAA mechanisms must be powerful as their intervention is often required. But even when flight trajectories are carefully planned in advance, many conflict situations can arise: high priority flights may force UAS to change courses, to decelerate or to stop for a moment and, in turn, further UAS have to react on these changes; unforeseeable events like strong wind gusts or mechanical failures can cause collision courses which have to be resolved in very short time; also flight corrections to avoid collisions with birds can result in further collision courses.

The DAA has two functions: "Detect" aims to get awareness about potential collision courses; "Avoid" provides correction maneuvers for all conflicting UAS. "Detect" gets the required information over communication links be it AA communication or AG communication, and from local sensors such as cameras, radars, and lidars. The communication messages contain information about the current position, current velocity vector, future waypoints, destination, priority mode, information about size, volume, freight type of a particular UAS. It is obvious that the communication link must be reliable and highly available. An interruption of the link is crucial. Furthermore, interference, jamming and spoofing is crucial. Further research on the robustness and availability of communication links considering a variety of hazards and appropriate countermeasures is very important.

Communication links have the advantage that they can be operated 24/7. For example, the recent remote ID rulemaking by FAA [13] require (through the standard remote ID capable drone option which is one of the three modes) that the drone broadcasts some critical information. Such information include the drone's unique ID, location and altitude, velocity, control station location and elevation, time mark, and emergency status. This broadcast message can be at a spectrum similar to what is used by Wi-Fi and Bluetooth, while the rule making does not specify or mandate a fixed frequency or technology. Such remote ID information, if can be reliably, seamlessly, and continuously monitored, will be the primary source for DAA operations. Additional research is needed to compare suitability of different wireless technologies for remote ID operations.

The RF signals between a drone and its controller can also be passively monitored for detecting, classifying, localizing, and tracking a drone [14]. This requires prior training of the system with signals from various commercial drones and their controllers. While various different machine learning algorithms are explored to improve detection and classification accuracy, there is need to reduce detection and classification errors especially at low SNRs. For improved tracking, there is further research needed to develop mobility models that consider typical operational environments and vehicle dynamics of typical drones and AAM vehicles.

Local sensors like cameras can be used complementary to communication links but require good visibility conditions, a high visual resolution, and sufficient on board computing power. Also radar and lidar can complement or backup communication links. Radars can be used to not only detect and track the drones, but also classify them using their measured radar cross section and additional microDoppler features [15]. There is further research needed for developing DAA mechanisms to distinguish birds from small drones, which can 
benefit from RCS and micro-Doppler based features. Local sensors may have difficulties to detect small obstacles and small UAS from larger distance. Landing of UAS on rooftops could be an ultimate option when communication is jammed, and other DAA modes are not effective.

When the DAA mechanisms identify that there is a collusion course involving one or more UAS, centralized (UAS action communicated by a remote entity) or UAS-centric (each UAS takes their own decision) measures can be taken. When there are collision courses where more than two UAS are involved, there is a need to develop novel avoid concepts. These situations occur when UAS fly on air corridors one after the other: when a UAV has to decelerate it has an impact on all other UAS following the first one - similar to platooning on roads for self driving vehicles. Correction courses may use the third dimension causing UAS to use air layers where no UAS is expected to fly. However, if in these air layers already other UAS are present, new conflicts may arise. With only a few UAS in operation this issue is not of great significance but will become crucial with a growing number of UAS. When collision avoidance maneuvers cause further conflicts, the overall stability of AAM is in danger.

\section{OTHER RESEARCH AREAS}

In this section, few other areas of research including: Navigation in GPS-denied environments; Noise mitigation; Security and Privacy; and Community Acceptance are outlined.

\section{A. Navigation in GPS-denied environments}

GPS signal is critical for a UAS to estimate its own location in the airspace. Without knowing its own location at any given time, a UAS may not be able navigate to its next intended location. Potentially a UAS may crash if the GPS signal is not available for more than few seconds, unless there is another strategy to estimate its location accurately. Although there are several alternatives based on cameras, inertial measurement units, and machine learning, such methods are not robust enough to substitute for GPS. A potential strategy may be a combination of such methods.

\section{B. Noise Mitigation}

Noise pollution is a major concern with UAS deployment in urban settings and it is barrier for to community acceptance of AAM [1], [16]. UAS may have radically different noise characteristics from that of traditional aircrafts due to differences in size, weight, and the technologies used in propulsion and airframe design. Strategies for noise mitigation include reducing engine noise, surface texturing, measurement, and modeling of acoustic footprint. The lack of descriptive aircraft models causes uncertainty in the estimation of acoustic signals. Accurate $3 \mathrm{D}$ vehicle profiles will improve the prediction of the noise response.

\section{Security and Privacy}

Although there are many benefits of UAV services, there are also potential vulnerabilities. For example,UAVs flying illegally may pose threats and danger to the human lives and infrastructure. Detecting such threats requires surveillance radars with the capability to detect, track, and measures of safely disengaging the rogue UAVs. There is also a need for encrypting and authenticating the information being shared by UAVs for mission-critical applications. Measures need to be in place for safety and security of people and for protecting their privacy when UAVs are flying over people.

\section{Community Acceptance}

Community acceptance is a critical factor to the success of AAM. Communities need to be informed of the changes to the transportation systems in their neighborhood. Education, training, outreach, and workforce development programs are the keys for gaining public trust in AAM. Government, public, private, and educational institutions need to come together to solve social challenges involved in making unmanned air transportation affordable, accessible, and equitable to one and all. When citizens appreciate that the benefits will outweigh the risks, communities will welcome the addition the new dimension of air mobility to their lifestyle.

\section{REFERENCES}

[1] National Academies of Sciences, Engineering, and Medicine. Advancing aerial mobility: A national blueprint. National Academies Press, 2020.

[2] Federal Aviation Authority. Urban Air Mobility: Concept of operations. Department of Transportation, 2020.

[3] M. Stevens and E. Atkins. Geofence definition and deconfliction for uas traffic management. IEEE Transactions on Intelligent Transportation Systems, pages 1-10, 2020.

[4] Wahab Khawaja, Ismail Guvenc, David W Matolak, Uwe-Carsten Fiebig, and Nicolas Schneckenburger. A survey of air-to-ground propagation channel modeling for unmanned aerial vehicles. IEEE Communications Surveys \& Tutorials, 21(3):2361-2391, 2019.

[5] John David Parsons. The mobile radio propagation channel. Wiley, 2000.

[6] David W Matolak and Ruoyu Sun. Air-ground channel characterization for unmanned aircraft systems-part iii: The suburban and near-urban environments. IEEE Transactions on Vehicular Technology, 66(8):6607-6618, 2017.

[7] Dennis Becker, Uwe-Carsten Fiebig, and Lukas Schalk. Wideband channel measurements and first findings for low altitude drone-to-drone links in an urban scenario. In Proceedings of the 14th European Conference on Antennas and Propagation, EUCAP '20. IEEE, 2020.

[8] 3GPP. Enhanced LTE support for aerial vehicles, Release 15. In TR 36.777, June 2018. Available at http://www.3gpp.org/.

[9] X. Lin, V. Yajnanarayana, S.D. Muruganathan, S. Gao, H. Asplund, H.-L. Määttänen, M. Bergström, S. Euler, and Y.P.E. Wang. The Sky Is Not the Limit: LTE for Unmanned Aerial Vehicles. IEEE Communications Magazine, 56(8):204-210, April 2018

[10] Ericsson. New WID on Enhanced LTE Support for Aerial Vehicles. In RP-172826, 2017. Available at http://www.3gpp.org/.

[11] Yong Zeng, Ismail Guvenc, Rui Zhang, Geraci Giovanni, and David W Matolak. UAV Communications for $5 G$ and Beyond. Wiley, 2020.

[12] Ericsson. New WID on Enhanced NR Support for Aerial Vehicles . In RWS-210189, 2021. Available at http://www.3gpp.org/.

[13] FAA. Remote identification of unmanned aircraft. Federal Register RIN 2120-AL31, Jan. 2021.

[14] Martins Ezuma, Fatih Erden, Chethan Kumar Anjinappa, Ozgur Ozdemir, and Ismail Guvenc. Detection and classification of UAVs using RF fingerprints in the presence of Wi-Fi and Bluetooth interference. IEEE Open Journal of the Communications Society, 1:60-76, 2019. Dataset: https://ieee-dataport.org/ open-access/drone-remote-controller-rf-signal-dataset.

[15] Martins Ezuma, Chethan Kumar Anjinappa, Mark Funderburk, and Ismail Guvenc. Radar cross section based statistical recognition of UAVs at microwave frequencies. Trans. IEEE Aerospace and Electronic Syst., 2021.

[16] Xinrong Li. Private Communication, 2021. 